\title{
Diverse Nanofibrous Silicon Structures Induced by Identical Plasma Energy Deposition
}

\author{
Chirag Paladiya, Amirkianoosh Kiani \\ Silicon Hall: Laser Micro/Nano Fabrication Facility, Faculty of Engineering and Applied Science, Ontario \\ Tech. University (UOIT) \\ Oshawa, ON, Canada \\ amirkianoosh.kiani@uoit.ca
}

\begin{abstract}
With identical extent of energy deposition, alteration in silicon fibrous structure was performed using variation in laserpower and scanning-speed. For validation and further comprehension, SEM analysis and reflective behavior of the surface were studied. Our results show that due to the unique nature of energy deposition by laser ablation process, we could generate relatively denser and defined fibrous structure in considerably shorter period of time at relatively higher laser power and scanning speed. In addition, by controlling the ablation condition, optical behaviors of the generated nanofibrous thin-film (light absorption and reflection) can be customized.
\end{abstract}

Keywords: Thin films, Antireflection coatings, Laser materials processing, Three dimensional fabrication, Nanostructure fabrication.

\section{Introduction}

Use of nano structured sensing surface is trending massively for new generation of electrical and photonic devices as described in our previous work with detailed literature review [1]. With significant efforts and available research, it is now possible to fabricate hybrid silicon nano fibrous structure at room temperature with the novel approach of laser ablation as already proven with our previous studies [2,3]. Furthermore, as described in our previous work along with experiments done by Tom et al. [4] and Stampfli et al. [5], energy deposition in terms of packets of concentrated photons with laser ablation is quite unique which provides freedom to vary the deposition sequence and intensity; even with the same extent of total energy input on the processed areas.

With certain specific laser and scanning parameters, it is possible to fabricate nano structured sensing surface consisting either nano fibers or nano particles and in some cases, combination of both [2]. However, in such cases, total energy deposited on the processed area varies significantly. By setting deposited energy on the machined area constant, irrespective of time required, one can change intensity of the ablation by varying power values and rate at which energy is being deposited by altering the value of scanning speed (SS). Employed setup and process parameters for this study are explained in the discussion below.

\section{Experimental setup and process parameters}

For this study, Ytterbium discontinues fiber laser (IPG Laser Model : YLPP-1-150V-30) along with wavelength of $1064 \mathrm{~nm}$ was employed for laser ablation of n-type silicon < $100>$ wafer. Laser parameters like pulse duration of $150 \mathrm{ps,}$ frequency of $1200 \mathrm{kHz}$ and pitch of $0.038 \mathrm{~mm}$ were kept constant. Constant energy deposition of $1.315 \mathrm{~kJ}$ was achieved on $10 \mathrm{~mm} \times 10 \mathrm{~mm}$ area of silicon by varying power and scanning speed shown as below. 
Table 1: Combination of laser parameters for identical energy deposition.

\begin{tabular}{|l|l|l|l|l|}
\hline Sample No. & Power $(\mathrm{W})$ & SS $(\mathrm{mm} / \mathrm{s})$ & Energy $(\mathrm{kJ})$ & Processing Time (s) \\
\hline S1 & 15 & 30 & 1.315 & 87.72 \\
\hline S2 & 20 & 40 & 1.315 & 65.79 \\
\hline S3 & 25 & 50 & 1.315 & 52.63 \\
\hline
\end{tabular}

\section{Results and Discussion}

Achieved results from this study was first studied to compare the topological structure formed with various laser and scanning parameters. SEM analysis of all three samples can be seen in Fig 1. Effect of intensity of laser is noticeable as the power was increased from $15 \mathrm{~W}(\mathrm{~S} 1)$ to $25 \mathrm{~W}(\mathrm{~S} 3)$. The structure generated with lowest power and scanning speed depicts the presence of both, nano particles and nano nano fibers. However, with the increased processing values, fibers appear to be much more defined and dense with no indication of silicon nano particles. In addition, as shown in Fig 2(a), it was possible to maintain deposited energy value of $1.315 \mathrm{~kJ}$ by proper combination of laser power and scanning speed. Also, our previous studies indicated increase in theoretical ablation temperature and elevated oxidation level with the increase in laser power level [2]. Hence, considering the similar trend followed in this study, oxidation level must increase with given conditions. Light spectroscopy of the processed samples can be helpful to identify the relative presence of oxidation level.

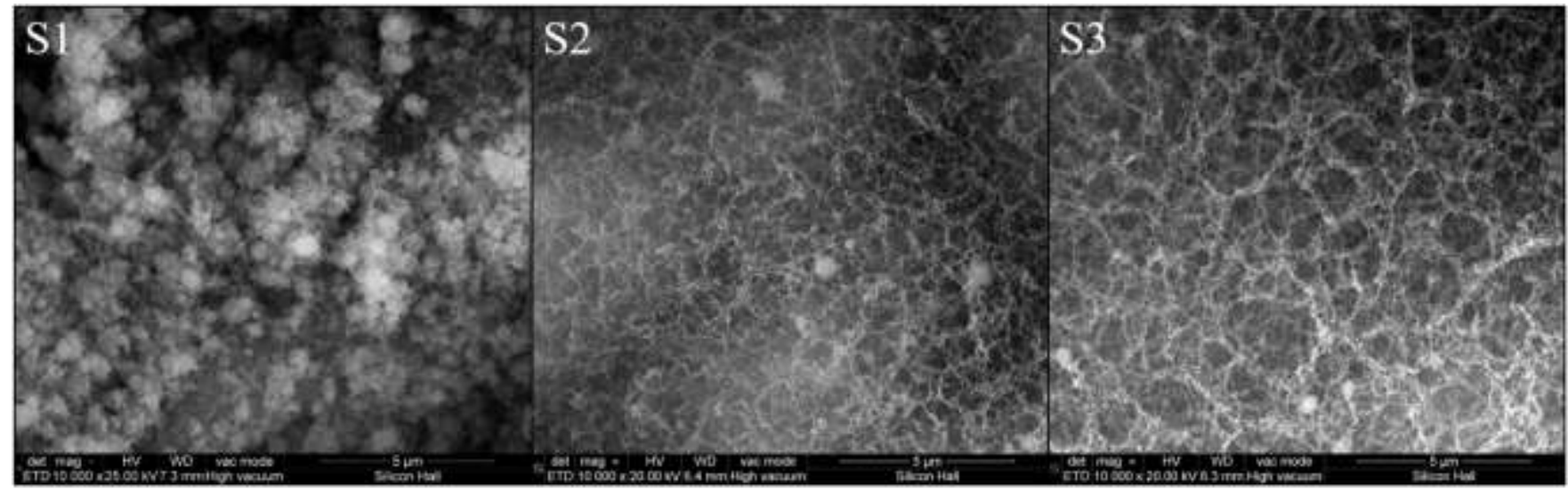

Fig. 1: SEM (Scanning Electron Microscopy) images of silicon samples.

The resulted structure shown in Fig 2(b) depicts slight change in color of processed samples with noticeable change in surface roughness, which can be seen with naked eyes. As the power was increased from $15 \mathrm{~W}$ to $25 \mathrm{~W}$, surface distortion happened because of higher ablation temperature can be seen with Fig 2(b). As indicated in our previous studies, uniform and higher ablation temperature contributes to better processing conditions causing better fiber generation possibilities, which can be confirmed with Fig 1. With increase in surface area, if the structure has similar oxidation level, reflection should decrease accordingly. Contrary, as shown in Fig 2(b), reflective behavior becomes dominant with the increased fiber density, which confirms the increased oxidation lever with elevated processing values. 
(a) Energy input combination

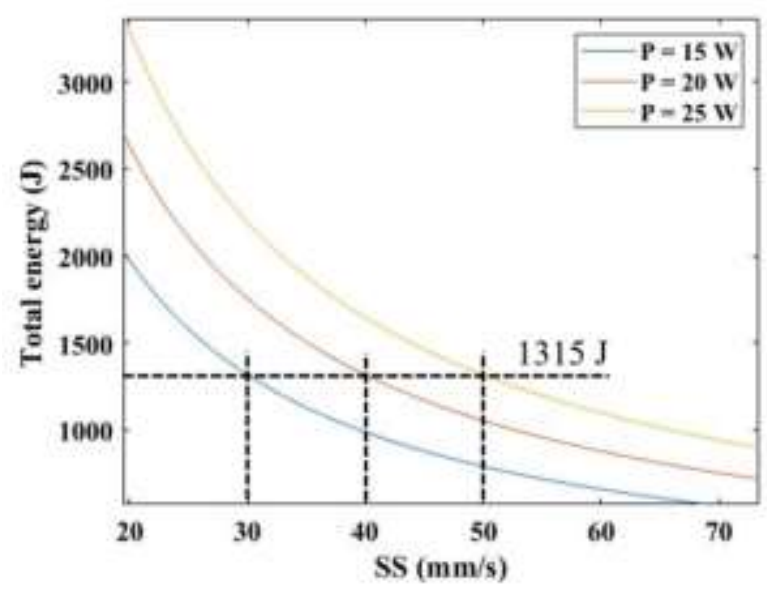

(b) Reflectivity of silicon samples

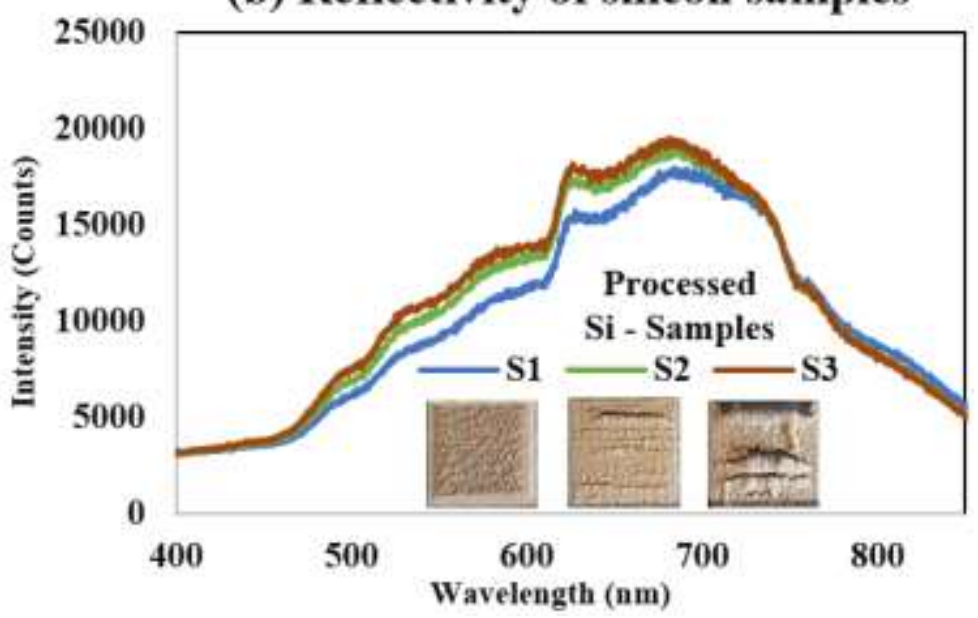

Fig. 2: (a) Energy input combination with laser power and scanning speed and (b) Light spectroscopy results.

\section{Conclusion}

In this study, importance of intensity of energy deposition irrespective of total amount of energy deposited on a silicon surface was understood. In addition, because of the unique nature of energy deposition by laser ablation process, with relatively higher laser power and scanning speed, it was possible to generate relatively denser and defined fibrous structure in considerably shorter period of time. Also, with favorable ablation condition caused by increase in selected processing values, relatively stronger reflective behavior was achieved. We strongly believe the findings in this research can lead to new solutions for advanced optical sensors and photonic applications.

\section{Acknowledgements}

This research was partially funded by the National Sciences and Engineering Research Council (NSERC).

\section{References}

[1] C. Paladiya, A. Kiani, "Nano structured sensing surface: Significance in sensor fabrication," Sensors and Actuators B: Chemical, vol. 268, no. 1, pp. 494-51, 2018.

[2] C. Paladiya, B. Kennedy, A. Kiani, "Pulsed ionized mesh-like assembly of hybrid silica nanostructures with controlled resistivity,” Appl. Surf. Sci., vol. 453, pp. 405-415, 2018.

[3] C. Paladiya, A. Kiani, "Synthesis of Graphite Embedded SiO2 Nanofibers with Controlled Conductivity," in Proceedings of IEEE for 2018 Photonics North (PN), Montreal, QB, Canada, 2018, vol. 1, pp. 1.

[4] H. W. Tom, G. D. Aumiller, C. H. Brito-Cruz, "Time-resolved study of laser-induced disorder of Si surfaces," Phys. Rev. Lett. E., vol. 60, no. 14, pp. 1438, 1988.

[5] P. Stampfli, K. H. Bennemann, "Theory for the instability of the diamond structure of Si, Ge, and C induced by a dense electron-hole plasma," Phys. Rev. B., vol. 42, no. 11, pp. 7163, 1990. 